\title{
Stochastic Capacity Acquisition and Allocation Model for Bandwidth Brokers under Fuzzy Volume Based Pricing Scheme
}

\author{
Hasan Hüseyin Turan ${ }^{1 *}$, Nihat Kasap ${ }^{2}$, Dursun Delen ${ }^{3}$ and Mehmet Nahit Serarslan ${ }^{4}$ \\ ${ }^{1}$ Department of Mechanical and Industrial Engineering, College of Engineering, Qatar University, Doha 2713, \\ Qatar \\ E-mail: hasan.turan@qu.edu.qa \\ ${ }^{2}$ Sabanci University, School of Management, Tuzla, 34956, Istanbul, Turkey \\ E-mail: nihatk@sabanciuniv.edu \\ ${ }^{3}$ Spears School of Business, Oklahoma State University, Oklahoma, USA \\ E-mail: dursun.delen@okstate.edu \\ ${ }^{4}$ Istanbul Technical University, Department of Industrial Engineering, Maçka, 34367, Istanbul, Turkey \\ E-mail: seraslann@itu.edu.tr
}

Received 8 December 2014

Accepted 1 January 2016

\begin{abstract}
In this paper, bandwidth acquisition and allocation problem of a telecommunications Bandwidth Broker (BB) is analyzed under uncertain end-user capacity requests and pay-per-byte (volume) based pricing policy. Furthermore, related objective function coefficients such as revenue and costs are modeled as fuzzy numbers in order to cope with vague market conditions. By integrating fuzzy mathematical programming and two-stage stochastic programming techniques, deterministic equivalent of single objective profit maximization problem of $\mathrm{BB}$ is obtained solved to optimality. In addition, infrastructure related performance measures such as delay and jitter amounts in the network are modelled via stochastic parameters that obey some known probability distributions. Two performance statistics namely fuzzy Expected Value of Perfect Information (EVPI) and fuzzy Value of Stochastic Solution (VSS) are defined to demonstrate the efficiency of proposed methodology compared to deterministic approach. In addition, several secondary performance measures such as expected capacity utilization, expected demand fulfilment ratio and capacity loss are calculated under different problem settings. In conclusion, numerical experiments showed that fuzzy stochastic method provides more profit depending upon problem size in compression with deterministic strategy.
\end{abstract}

Keywords: Telecommunications market, bandwidth broker, fuzzy stochastic mathematical programming, fuzzy VSS and EVPI

\section{Introduction and Motivations}

During the last decades with the increasing usages of internet and other communication tools, telecommunications networks have become a very complex environment. It is possible to classify decision making problems that are encountered in telecommunication environment into three different levels such as technological, network and enterprise ${ }^{1,2}$. Fig-

\footnotetext{
* Corresponding author: E-mail:hasan .turan@qu . edu .qa; Tel: (+974) 3309-0921; Fax: (+974) 4403-4101.
} 
ure 1 depicts telecommunication problem topologies. The technological level deals with design of different elements of telecommunication networks such as routers and switches ${ }^{2}$, packet scheduling and buffer management problems. Network level problems concentrate on design and planning of different kinds of telecom networks which may differ by size and by technology involved. Several decisions such as capacity expansion decision of links and allocation of servers are dynamic in nature and consist of several time periods. At the enterprise level telecom firms are considered as an actor in a market where they interact with other actors such as industrial companies and customers; thus this level can be classified as integration at the highest level. Determination of pricing policies, capacity provider selection and resource allocation, and selection of service types that telecom firm will offer to the market are examples of some basic decisions taken at this level ${ }^{2,3}$.

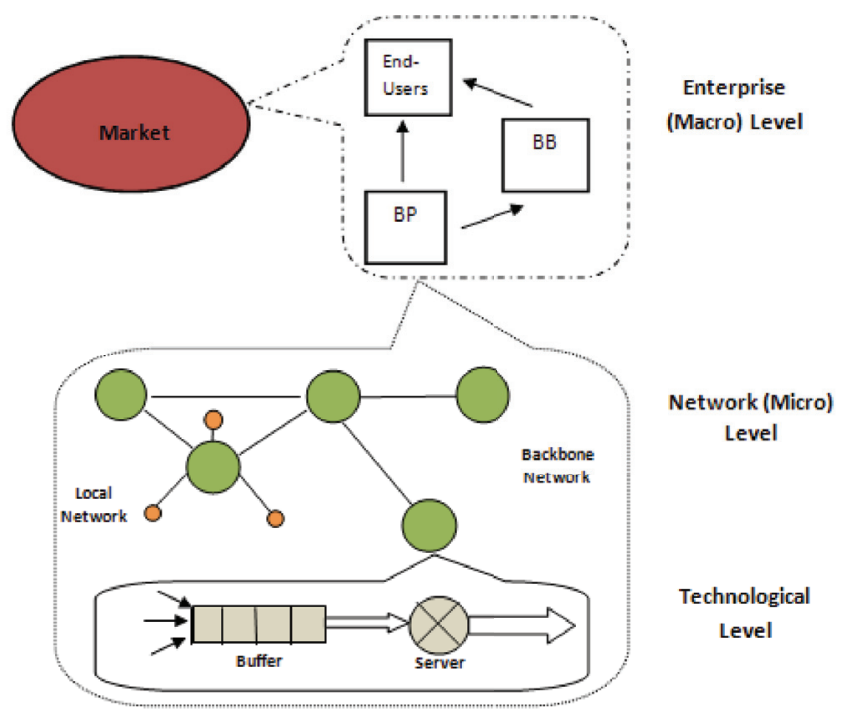

Fig. 1. Telecommunications' problems topology ${ }^{1}$.

The problem studied in this research falls into the enterprise level category. At this level, it is assumed that telecommunications market consists of three important main players, which are customers or end- users who use network capacity to accomplish their tasks, backbone network providers (BP) and intermediaries or bandwidth brokers (BB). Whereas BPs own the network infrastructure, BBs do not have their own network facilities and in order to provide the service BBs have to lease the necessary network capacity from BPs or other bandwidth brokers. Consequently, BBs have to decide how much capacity to acquire and the BPs decide the price to charge for the network capacity ${ }^{3}$.

Due to the introduction of new technologies and services such as GPRS in GSM or 'video on demand' for digital subscriber lines, which have led to increase in the end-customer demands ${ }^{4}$. Figure $2^{\dagger}$ depicts the progress of number of broadband users in Turkish telecom market. Hence, with the increasing number of end-customers, number of internet service providers (ISP) has increased in the market as well, which led to higher completion among ISPs.

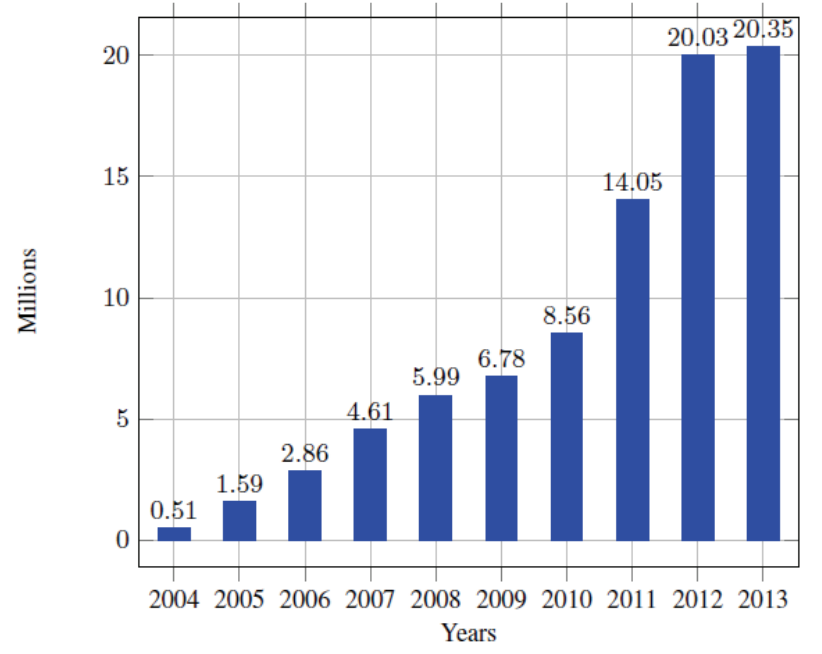

Fig. 2. Number of Broadband Internet Subscribers in Turkey.

In Figure $3 ;$ market shares of Turkish IPSs in the recent years are presented. It can be concluded from recent data that even though market leader TTNet (only backbone in the market) keeps its position, its share decreases steadily. On the other hand, shares

\footnotetext{
$\dagger$ Retrieved from http://eng.btk.gov.tr/kutuphane_ve_veribankasi/pazar_verileri/2013_Q1_ECM_MarketData.pdf on $14 / 02 / 2014$.

$\ddagger$ Retrieved from http://webrazzi.com/2014/03/24/ulkemizdeki-internet-servis-saglayicilarin-pazar-paylari \-webrazzi-pro/ on 24/10/2014.
} 
of BBs such as Superonline and Doğan Diğital increase in total. Therefore, modeling and solution strategies are needed for BBs in order to stay competitive in emerging markets, which is one of the motivations of this study.

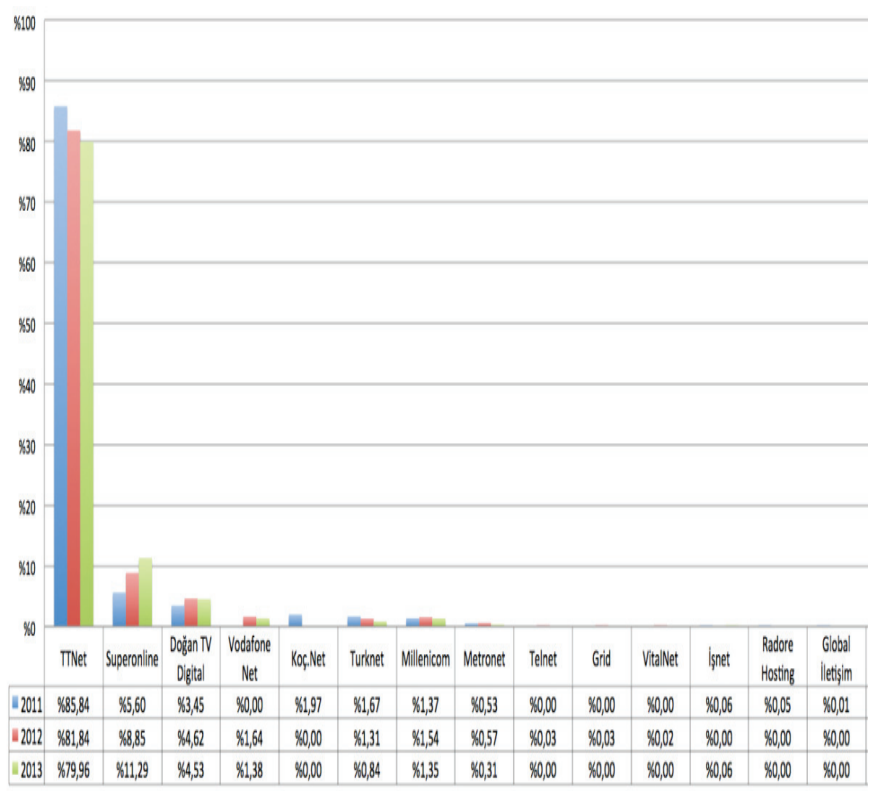

Fig. 3. Progress of ISPs' market shares in Turkey.

We consider BB's profit maximization problem in an environment in which the firm can lease network capacity at competitive prices from different BPs with different quality of service (QoS). In order to gain profit, a BB has to first acquire (lease) the capacity (bandwidth) from BPs then lease bandwidth to end-users. After leasing decision, the BB has to allocate leased bandwidth into acquired capacities by meeting QoS requirements of each customer. Figure 4 depicts relationships and interactions between each players in the modeled telecommunications market.

The solution of the proposed model provide essential strategic planning information to the decision makers of BB such as how much bandwidth for how long should be leased from each BP, which service providers should be chosen by considering QoS levels, after realization of customers' demands which customer's bandwidth request should be accepted and which of them should be rejected. In addition, solution provides information about how accepted bandwidth demands should be allocated into leased capacity by considering quality of service parameters such as delay and jitter.

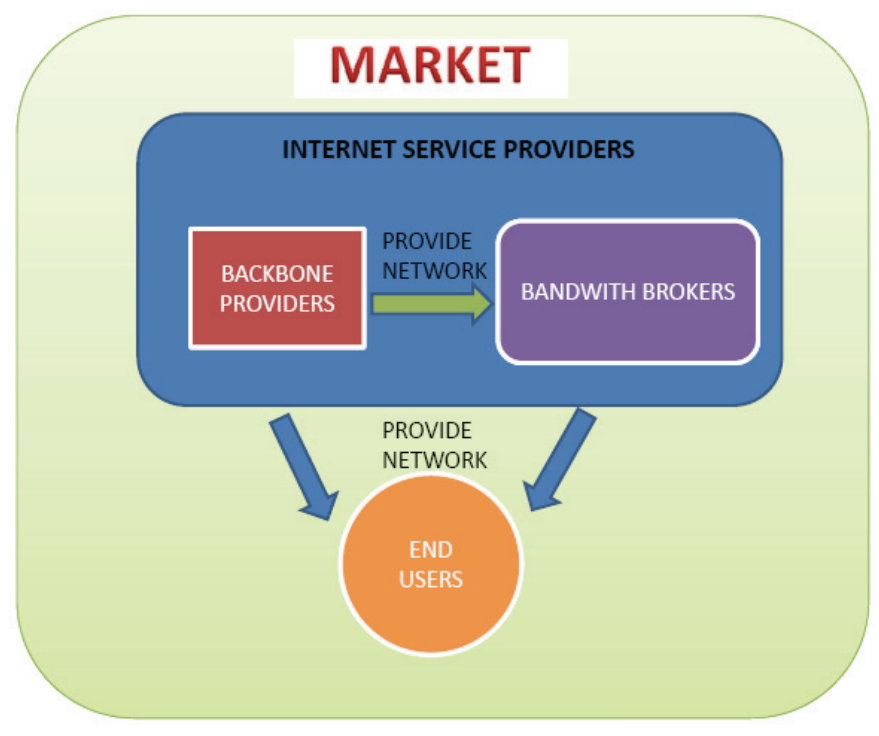

Fig. 4. The modelled market structure.

Like all real world phenomena, telecommunication network are affected by uncertainties. Uncertainties in telecommunications may arise from either environmental or internal reasons. In either case, neglecting uncertainties in the modelling stage may result in inaccurate and inefficient solutions ${ }^{1}$. Hence, we model and solve bandwidth acquisition and allocation problem of a telecommunications BB by taking into account uncertainties arising due to market and infrastructure conditions and by integrating fuzzy and stochastic programming methodologies.

In the remaining of this section, brief background information about pricing policies and QoS parameters in telecommunications are provided which are required to build up BB's profit maximization model. Afterwards contributions and uniqueness of this study are emphasized.

There exist several pricing policies to model capacity acquisitions between BB and BPs as well as BB and end-users. Two widely used classes of pricing policies are static pricing and dynamic pricing. In the static pricing schemes, prices are fixed and are not depended on the system's state. On the other hand, in dynamic pricing schemes, the service provider sets prices to the consumers based on 
the value; i.e., the amount of data, the transmission rate and Quality of Service (QoS) that the customers consider to the lease. Dynamic schemes models are believed to be more powerful and flexible than static pricing strategies and have been used in many areas of industry in addition to telecom (see rf. ${ }^{5,6,7}$ ), such as retailing, manufacturing, airlines, and ebusiness $^{8,9}$. To illustrate, well-known all-you-cansend (or flat rate) pricing policy is a static scheme in which the customer leases bandwidth at a fixed price for a fixed contract period ${ }^{10,11}$. On the contrary, the pay-per-byte or volume based pricing scheme is a dynamic scheme due to the fact that total usage cost is calculated as a function of data transmitted without any loss which highly depends on the network conditions such as instantaneous congestion during data transmission ${ }^{12,13}$. A brief summary of past researches on internet pricing and dynamic pricing models can be found in ${ }^{14}$. In this research, the payper-byte scheme is integrated into profit maximization objective of BB to model both acquisition costs occurring during capacity purchases from BPs and capacity selling revenues obtained during bandwidth leasing to end-users. Applying volume based pricing scheme during both capacity buying and selling stages can be considered as one of the uniqueness of proposed model.

Serving numerous users with highly-diverse contents under limited resource capacity is a challenging issue ${ }^{15}$. Therefore, end-users with different requirements are classified into different service classes by capacity providers. For each class a particular QoS is assigned, which defines group of service measures representing the terms of user agreement of the service is assigned. Several metrics are defined in literature to measure QoS. Figure 5 presents a comprehensive taxonomy created by Malik et al. ${ }^{16}$. Common QoS metrics used during service provisioning are delay, packet loss, jitter, response time, reliability, and availability. Delay is measured as travel time of data packet from source to destination across the network structure ${ }^{17,18,19}$. The delays of scheduling from data packet to data packet may lead to buffered packet delay because of altering delays in transmission. This phenomenon is known as jitter ${ }^{20}$. The packet losses occur due to buffer overflow and indicates data dropped or damaged ${ }^{1}$. OoS requirements are usually applicationspecific. Certain applications such as voice conferencing are delay and jitter sensitive. For example, for VoIP applications round-trip delay must be in the range of to $200-300 \mathrm{~ms}$, and jitter has to be less than $50 \mathrm{~ms}$ so that playback at the receiver remains smooth ${ }^{21}$. In general, interactive applications, such as web browsing, video conferencing and live streaming are delay and jitter sensitive. On the contrary, Email, file sharing and remote login are not affected by jitters in the network traffic ${ }^{16}$. In addition, routine operations usually performed by firms such as bulk file transfer are concerned more with average packet loss rate. In this study, we will only focus on the QoS parameters of delay, jitter, and packet loss rate. In conclusion, BBs and all ISPs must support multiple QoS strategies to support different applications. However, without a appropriate pricing strategy, any QoS based scheme would be useless. Thus, BB has to identify suitable price for each end-user class and then allocate necessary network resources to meet all service agreements.

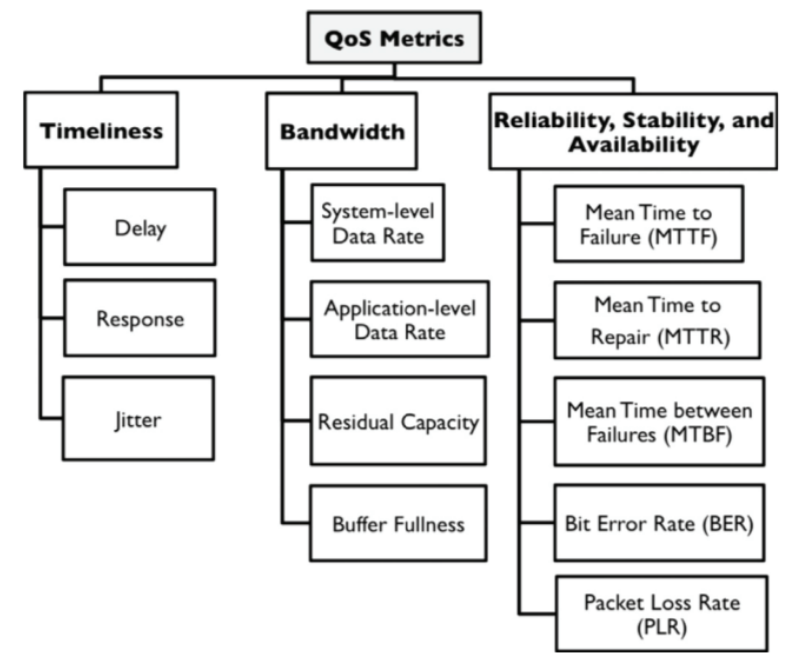

Fig. 5. Classification of QoS metrics ${ }^{16}$.

The proposed research contributes the existing literature in both managerial and theoretical perspectives as follows: (a) BB's point of view modeling approach rather than provider or firm side modeling, which dominates the current telecommunications literature. (b) Taking into account mar- 
ket related uncertainties such as profit and cost terms via fuzzy methodology and handling infrastructure related unknowns such as QoS parameters by stochastic programming techniques. (b) Integration fuzzy and stochastic techniques for solving managerial (macro) level telecommunication problem rather than network infrastructure (micro) level such as routing and network topology design problems. (d) From algorithmic point of view, development of two new metrics fuzzy expected value of perfect information and fuzzy value of stochastic solution in order to measure efficiency of methodology compared to deterministic approaches.

The rest of the paper is organized as follows: Section 2 presents brief literature survey about non-deterministic methodologies that are applied to telecommunication network problems both on managerial and infrastructure levels. Section 3 provides background information about fuzzy and stochastic programming then discusses how these two methodologies are integrated in order to solve BB's profit maximization problem. In this section, the proposed model with probabilistic QoS constraints is discussed. In Section 4, algorithms for main performance statistics are provided. In addition, an extensive computational study with sensitivity analysis is given in order to demonstrate the efficiency of suggested method in comparison with deterministic approaches. Finally, Section 5 summarizes important results and describes future goals respectively.

\section{Related Works}

Stochastic and fuzzy methods have been applied to telecommunications problem for decades in order to find optimal or near optimal solutions under uncertainty. Hence, there exists huge amount of publication in the current literature. Related literature is divided into subsections to classify methods developed to solve different level of telecommunication problems. The first part of literature review lists article related to supplier selection and demand allocation not only in telecom networks but also in supply chain literature. In the following part, articles related to the fuzzy based routing algorithms in the networks are presented. The remaining parts are devoted to modeling of QoS parameters under uncertainty and forecasting of demand with fuzzy based methodologies. Besides, detailed discussions and review papers about fuzzy logic applications in telecommunications can be found in refs. ${ }^{22,23,24}$.

The first stream of the literature survey concentrates on provider (supplier) selections and order (demand) allocation articles encountered in telecommunications and the supply chain. Turan et al. ${ }^{25}$ investigate a stochastic optimization problem that a telecommunications intermediary faces when acquiring network capacity from a market. The problem is modeled as a two-stage stochastic integer programming under all-you-can send pricing policy. On the other hand, QoS parameters are assumed to be deterministic and modeled via crisp constraints. Finally, optimal behavior of intermediary analyzed under different problem settings such as changing demand distribution type and variance of the demand distribution of end-users. In studies ${ }^{26}$ and ${ }^{3}$ QoS parameters (delay and jitter) are modeled as random variables, in which end-users and intermediaries are considered as decision makers, respectively. On the other hand, delay and jitter amounts guaranteed by network providers and maximum delay jitter levels tolerable for end-users are modeled as fuzzy numbers from telecom firm's point of view in ${ }^{27}$. An outsourcing provider selection problem for a telecommunication company under vague information is studied by Uygun et al. ${ }^{28}$. Their integrated method uses Fuzzy Analytic Network Process (FANP) technique in combination with Decision Making Trial and Evaluation Laboratory (DEMATEL) technique. In proposed method as a first step, DEMATEL method is used in to put forward the interrelationship among the main criteria (competence of the company in field projects, experience of the company, etc.), and then local weights of the sub-criteria (credibility, total capital, etc.) and subsubcriteria (GSM backgroud of managers, employee number in different units' etc.) are calculated by Fuzzy ANP approach on the basis of cause-effect relationships that are exposed through DEMATEL method. In addition, Fu et al. ${ }^{29}$ also come up with fuzzy DEMATEL based method to evaluate telecommunications system providers. They intro- 
duce a fuzzy (grey)-based DEMATEL approach that involves the identification of GSDPs, understanding their interrelationship utilizing "fuzzy" data input from management, transforming the fuzzy data into crisp data, completing a series of DEMATEL steps, and eventually arriving at a final prominence-causal relationship diagram with associated analysis. Another interesting outsourcing problem in ISP market analyzed by fuzzy multi-criteria method is presented in ${ }^{30}$. They use an interactive group decisionmaking methodology to select/rank IS providers under multiple criteria. The proposed group decision making approach heavily depends on fuzzy TOPSIS approach. They list seven criteria for IS provider selection such as price, product quality, on-time delivery, technological capability, quality of relationship with ISP, professionalism of salesperson, responsiveness to customer needs. Onut et al. ${ }^{31}$ present an integrated FANP and fuzzy TOPSIS (FTOPSIS) methodology to evaluate and select the most suitable suppliers for a telecommunication company in GSM sector. They use triangular fuzzy numbers in all pairwise comparison matrices, which is different from the conventional FANP method. In the mentioned study, criteria (cost, references, quality of the product, delivery time, institutionality, execution time) weights are inserted to the FTOPSIS methodology to rank the suppliers for selection.

In addition to telecommunications, Provider (vendor) selection and demand allocation problems under uncertainty especially under presence of vague information are studied extensively in the supply chain literature and several solution algorithms are proposed. Therefore, in this section of literature review a brief survey about fuzzy mathematical programming applications in other application domains rather than telecom is provided. Kabak et al. 32 propose a possibilistic linear programming (PLP) model for supply chain networking decision. In the investigated model not only problem parameters such as demands and cost terms but also decision variables such as production amounts are assumed to be fuzzy numbers. As a solution methodology, the PLP model is converted to an LP model by using mathematical operations defined for fuzzy numbers. Mula et al. ${ }^{33}$ investigate a material requirements planning problem in an industrial environment under lack of knowledge in data and existent fuzziness jointly in the processes of production planning. Their model considers fuzzy constraints related to the total costs, the market demand and the available capacity of the productive resources and fuzzy coefficients for the costs due to the backlog of demand and for the required capacity. There also exists vast amount literature about multi-objective fuzzy optimization for supplier selection and demand allocation problems in diverse areas such as $34,35,36$.

Another application of fuzzy logic in telecommunications is designing and configuring large networks. Douligeris 37 suggests using hybrid systems integrating expert systems and neural networks methods rather than classical hard decision methods for network design. Author presents an integrated fuzzy expert system, machine learning, and neural networks approach to evaluate computer network design. After designing stage, efficient routing decision and algorithms are needed. In this stage, a lot of critical routing factors should be taken into considerations such as reliability and availability. Fuzzy routing algorithms are commonly used alleviate arising difficulties in routing. To illustrate, Wang 38 claim that a fuzzy-based routing scheme based on reputation, bandwidth and distance can improve the throughput of network. The task of routing data is also investigated by Ortiz et al. ${ }^{39}$, in which fuzzy logic is utilized to perform role assignment during route establishment and maintenance. Number of hops to the base station, and the remaining node energy are considered as input variables and modeled as fuzzy number. At the implantation of algorithm, they use Mamdani-based fuzzy rules.

During routing decisions, QoS constraints have to be taken into account. Thus, routing algorithms used should avoid unexpected delays that result in QoS fluctuations. Fernandez et al. ${ }^{40}$ develop fuzzy control method to provide QoS guarantees in a DiffServ telecom network. Their methodology applies a genetic algorithm to traffic statistics to obtain fuzzy controller parameters. Afterwards, fuzzy controller parameters are optimized by a genetic algorithm in order to produce an improvement in QoS metrics such as delay and jitter for time-sensitive traf- 
fic. There are also some studies in which QoS parameters are modeled as fuzzy number. For example, Mansouri et al. ${ }^{41}$ propose a combined QoS and Quality of Experience (QoE) evaluation system by utilizing the combination of fuzzy inference systems and fuzzy evidence (Dempster-Shafer) theory. They define fuzzy trapezoidal membership functions, which are estimated by interviewing with networking experts, for each QoS metric such as response time, availability, jitter and bandwidth. Another research study proposed by Chen et al. ${ }^{42}$ also models QoS parameters as trapezoidal fuzzy number. However, other source of uncertainties regarding bandwidth requests or prices are not included in their model.

One of the vital inputs of network traffic management, infrastructure optimization and planning in telecommunications is forecasting the data (demand) traffic. Decision makers of BPs and BBs can predict demand by making use of the historical data. In addition to commonly used statistical forecasting techniques, computational intelligencebased approaches have pervaded recent literature. In this direction, Mastorocostas and Hilas ${ }^{43}$ propose a modified Takagi-Sugeno-Kang fuzzy neural system in order to forecast the outgoing call volume of a University Campus with more than 6000 employees and 70,000 students. They also include performance comparison of proposed methodology with seasonally adjusted linear extrapolation methods, exponential smoothing methods and the SARIMA method. Another fuzzy neural network based data volume forecasting method in telecommunications is suggested in ${ }^{44}$. It is claimed that mentioned method perform forecasting without requiring prior knowledge of the exact order of the time-series due to the consequent parts of the fuzzy rules being small block-diagonal recurrent neural networks with internal feedback. After successful forecasting of endusers demand, BBs as well as BPs have to look for solutions to keep their customers satisfied and thus loyal with the company. As a solution to this issue, Keropyan and Gil-Lafuente ${ }^{45}$ present a fuzzy based Hungarian method that allow assigning different loyalty programs to customers with different characteristics in order to provide a model that helps decision makers deciding what kind of customer loyalty programs they should offer to their clients from different segments. Hanafizadeh and Mirzazadeh ${ }^{46}$ also study market segmentation problem of a telecommunication company. They present a technique which integrates Fuzzy Delphi method and self-organizing maps (SOM) to cluster customers according to their various characteristic variables such as demographics, socio-economic factors, and geographic location.

A recent fuzzy c-means based segmentation (clustering) algorithm for telecommunication data is developed in ${ }^{47}$. Country level telecommunications issues analysed by fuzzy methods are investigated in 48,49 . To illustrate, Stula et al. ${ }^{48}$ develop a Fuzzy cognitive map (FCM)that is a qualitative modelling and behavior simulation method predicts Internet spreading rate in different countries. Another example can be found in ${ }^{49}$, in which data envelopment analysis (DEA) and fuzzy data envelopment analysis (FDEA) are integrated for efficiency assessment of wireless communication sectors. The proposed algorithm uses indicators as cellular cost of a 3-min local call, cellular connection charge, and mobile communication revenue as input for efficiency assessment and optimization. Finally, fuzzy models regarding to ranking of the strategic actions of mobile cellular telecommunication using a fuzzy quality function deployment (QFD) method is suggested by Khademi-Zare et al. ${ }^{50}$. They model QFD input parameters such as customer attributes (delivery time, sim card price etc.) and strategic actions (traffic measurement, information technology etc.)as fuzzy triangular numbers to overcome ill-defined judgments on inputs.

In conclusion, even though there exist several research articles related to application of fuzzy and stochastic programming in the telecommunications as well as other areas, there is almost no study that combines both methodologies neither at lower level (routing and design) nor at upper level (provider selection) communication networks areas. Thus, the proposed study can extend the use of fuzzy and stochastic programming in backbone selection and demand allocation under volume based pricing scheme. 


\section{Fuzzy stochastic linear programming model}

A fuzzy linear programming (FLP) model can be written as in Eq.(1) by using definition and notations introduced by ${ }^{51}$.

$$
\begin{array}{ll}
\text { Maximize: } & <\widetilde{c}, x>_{F}=\sum_{i=1}^{n} \widetilde{c}_{i} x_{i}, \\
\text { Subject to: } & A x \leqslant b, x \geqslant 0,
\end{array}
$$

where $<>$ operator denoting the scalar multiplication of vectors. $\widetilde{c}=\left(\widetilde{c}_{1}, \widetilde{c}_{2}, \ldots, \widetilde{c}_{n}\right)$ represents array of fuzzy numbers. $A$ is a matrix with dimensions $m \times n$ and $b \in R^{m}$ is a vector.

We assume that all fuzzy numbers used in the mathematical model have triangular membership function. In other words, any objective function coefficient can be redefined as $\widetilde{c}_{i}=\left(c_{i}^{L}, c_{i}^{M}, c_{i}^{U}\right)$. The linear multi-objective programming equivalent of model (1) is given in Eq.(2).

Maximize: $\quad\left(<c^{L}, x>,\left\langle c^{M}, x\right\rangle,\left\langle c^{U}, x>\right)^{T}\right.$, Subject to: $\quad A x \leqslant b, x \geqslant 0$,

Following is the single objective counterpart of the model (2):

$$
\begin{array}{cl}
\text { Maximize } & <w, \widetilde{c}, x>=\left(w_{1}<c^{L}, x>+\right. \\
& \left.w_{2}<c^{M}, x>+w_{3}<c^{U}, x>\right), \\
\text { Subject to: } & A x \leqslant b, x \geqslant 0,
\end{array}
$$

In model (3), $w$ is defined as $w=\left(w_{1}, w_{2}, w_{3}\right) \geqslant$ 0 . Model turns into parametric linear programming problem, if $w$ is chosen as $w_{1}+w_{2}+w_{3}=1$ and set to $w_{3}-w_{1}=\varepsilon$. Here, $\varepsilon$, has to be altered between -1 and $1^{51}$. All of the sets, parameters and decision variables used in mathematical formulation are presented in Table 1. The objective function of volume based pricing policy with fuzzy coefficients is presented in Eq. (4). Fuzzy revenues and fuzzy opportunity costs depend on scenarios therefore, the expected values for these terms have to be calculated. The fuzzy expected costs/revenues are obtained after mentioned terms are multiplied by realization probability of each scenarios $p_{s}$ and summed all over possible scenario set. The problem of $\mathrm{BB}$ is to maximize fuzzy expected profit under volume based pric-

\begin{tabular}{|c|c|}
\hline Index Sets & \\
\hline$I$ & Set of telecommunications BPs \\
\hline$\Omega$ & Set of scenarios that may occur \\
\hline$J_{s}$ & $\begin{array}{l}\text { Set of end-users under scenario } s \\
(s \in \Omega)\end{array}$ \\
\hline \multicolumn{2}{|l|}{ Parameters } \\
\hline$d_{j s}$ & $\begin{array}{l}\text { Amount of bandwidth request for } \\
j \text {.end-user under scenario } s\end{array}$ \\
\hline$\widetilde{\vartheta}_{j s}$ & $\begin{array}{l}\text { Fuzzy revenue earned by meeting } \\
j \text {.end-user's demand under scenario } s\end{array}$ \\
\hline$\widetilde{v}_{j s}$ & $\begin{array}{l}\text { Fuzzy opportunity cost for not meeting } \\
j \text {.end-user's demand under scenario } s\end{array}$ \\
\hline$p_{s}$ & Realization probability of scenario $s$ \\
\hline$\alpha_{i}$ & Capacity loss ratio for BP $i\left(\alpha_{i} \in[0,1]\right)$ \\
\hline$\widetilde{c}_{i}$ & $\begin{array}{l}\text { Fuzzy unit bandwidth cost for leasing } \\
\text { capacity from BP } i\end{array}$ \\
\hline$U_{i}$ & $\begin{array}{l}\text { Maximum amount of bandwidth } \\
\text { that can be leased from BP } i\end{array}$ \\
\hline$\Phi_{i}, \Psi_{i}$ & $\begin{array}{l}\text { Random variables to denote delay and } \\
\text { jitter distribution of BP } i \text {, respectively }\end{array}$ \\
\hline$\mu_{i}^{\delta}, \sigma_{i}^{\delta}$ & $\begin{array}{l}\text { Mean and standard deviation values } \\
\text { corresponding to delay random variable } \\
\text { of BP } i \text {, respectively }\end{array}$ \\
\hline$\mu_{i}^{\rho}, \sigma_{i}^{\rho}$ & $\begin{array}{l}\text { Mean and standard deviation values } \\
\text { corresponding to jitter random variable } \\
\text { of BP } i \text {, respectively }\end{array}$ \\
\hline$\Theta_{j s}^{\delta}, \Theta_{j s}^{\rho}$ & $\begin{array}{l}\text { Minimum required service level } \\
\text { probabilities for delay and jitter } \\
\text { measures in order to fulfill } j \text {.end-user } \\
\text { demand under scenario } s \text {, respectively }\end{array}$ \\
\hline$\delta_{j s}, \rho_{j s}$ & $\begin{array}{l}\text { Maximum tolerable delay and jitter } \\
\text { amounts for end-user } j \text { under scenario } s \text {, } \\
\text { respectively }\end{array}$ \\
\hline$\pi$ & Minimum required demand fulfillment ratio \\
\hline$\Pi_{\vartheta_{j s}}, \Pi_{v_{j s}}$ & $\begin{array}{l}\text { Membership functions for revenue and } \\
\text { opportunity, respectively }\end{array}$ \\
\hline$\Pi_{c_{i}}$ & Membership functions for leasing costs \\
\hline \multicolumn{2}{|l|}{$\begin{array}{l}\text { Decision } \\
\text { variables }\end{array}$} \\
\hline$\beta_{i}$ & Amount of bandwidth leased from BP $i$ \\
\hline$y_{i j s}$ & $\begin{array}{l}\text { The Proportion of bandwidth demand } \\
\text { of } j \text {.end-user allocated into BP } i \text { under } \\
\text { scenario } s\end{array}$ \\
\hline$y_{j s}$ & $\begin{array}{l}\text { Total proportion of bandwidth demand } \\
\text { of } j \text {.end-user meet under scenario } s\end{array}$ \\
\hline
\end{tabular}

ing scheme. New model is a fuzzy stochastic linear programming model (FSLP) with fuzzy objective parameters.

Table 1. Mathematical model notations. 


$$
\text { Maximize } \underbrace{}_{\text {expected fuzzy revenue }} \underbrace{\sum_{s \in \Omega} \sum_{j \in J_{s}} p_{s} \widetilde{\vartheta}_{j s} y_{j s}}_{\text {expexcted fuzzy oppurtunity cost }}-\overbrace{\sum_{i \in I} \beta_{i} \widetilde{c}_{i}}^{\text {fuzzy leasing cost }}-
$$

Eq.(5) explains how function $\Xi$ is evaluated in terms of problem parameters.

$$
\begin{aligned}
& \Xi\left(w_{1},\left\{\vartheta^{L}, c^{L}, v^{L}\right\}\right)=w_{1}\left(\sum_{s \in \Omega} \sum_{j \in J_{s}} p_{s} \vartheta_{j s}^{L} y_{j s}-\right. \\
& \left.\sum_{i \in I} \beta_{i} c_{i}^{L}-\sum_{s \in \Omega} \sum_{j \in J_{s}} p_{s} v_{j s}^{L}\left(1-y_{j s}\right)\right)
\end{aligned}
$$

After applying the methodology suggested by ${ }^{51}$ on objective function of BB in Eq. (4), transformed linear objective equation is showed in Eq.(6). Where unit revenues $\left(\widetilde{\vartheta}_{j s}=\left(\vartheta_{j s}^{L}, \vartheta_{j s}^{M}, \vartheta_{j s}^{U}\right)\right)$ and unit costs $\left(\widetilde{v}_{j s}=\left(v_{j s}^{L}, v_{j s}^{M}, v_{j s}^{U}\right)\right)$ and $\left(\widetilde{c}_{i}=\left(c_{i}^{L}, c_{i}^{M}, c_{i}^{U}\right)\right)$ are modeled as triangular fuzzy number (TFN). In computational experiments, different $w$ values are selected and run to provide useful statics to decision makers of BB.

$$
\begin{aligned}
& \text { Maximize } \Xi\left(w_{1},\left\{\vartheta^{L}, c^{L}, v^{L}\right\}\right)+ \\
& \Xi\left(w_{2},\left\{\vartheta^{M}, c^{M}, v^{M}\right\}\right)+\Xi\left(w_{3},\left\{\vartheta^{U}, c^{U}, v^{U}\right\}\right)
\end{aligned}
$$

Eqs.(6-15) presents FSLP model constraints. Constraint set (6) guarantees that it is not possible to allocate total customer demand that is more than the purchased capacity from corresponding BP under each scenario. Total capacity allocated to a particular backbone has to smaller than or equal to the acquired bandwidth from corresponding supplier minus capacity losses. Eq.(7) ensures that amount of bandwidth purchased from any BP can not be more than BPs' capacity that is sold at the market.

$$
\begin{aligned}
& \sum_{j \in J_{s}} y_{i j s} d_{j s} \leqslant\left(1-\alpha_{i}\right) \beta_{i}, \forall i \in I \forall s \in \Omega \\
& \beta_{i} \leqslant U_{i}, \forall i \in I
\end{aligned}
$$

Eq.(8) guarantees that bandwidth request of any end-user under any scenario can only be assigned to a purchased capacity that satisfies delay requirement of user above some predefined probability.

$$
\mathbb{P}\left\{y_{i j s}\left(\Phi_{i}-\delta_{j s}\right) \leqslant 0\right\} \geqslant \Theta_{j s}^{\delta}, \forall s \in \Omega, \forall i \in I, \forall j \in J_{s}
$$

Similarly, Eq.(9) ensures that bandwidth demand of any end-user under any scenario can only be allocated to a particular purchased capacity that meets jitter requirement of customer above predefined probability.

$$
\mathbb{P}\left\{y_{i j s}\left(\Psi_{i}-\rho_{j s}\right) \leqslant 0\right\} \geqslant \Theta_{j s}^{\rho}, \forall s \in \Omega, \forall i \in I, \forall j \in J_{s}
$$

It is generally assumed that QoS parameters are distributed normally. Based on this assumption, deterministic counter parts of Eqs.(8) and (9) can be rewritten as in Eqs.(10) and (11), respectively. $\Gamma_{\Theta_{j s}^{\delta}}$ and $\Gamma_{\Theta_{j s}^{\rho}}$ denote quartile function values for standard normal distribution.

$$
y_{i j s}\left(\frac{\delta_{j s}-\mu_{i}^{\delta}}{\sigma_{i}^{\delta}}-\Gamma_{\Theta_{j s}^{\delta}}\right) \geqslant 0 \forall s \in \Omega, \forall j \in J_{s}, \forall i \in I
$$

Both inequalities are in linear form, so FSLP of $\mathrm{BB}$ can be solved via traditional linear programming texhniques such as simplex algorithm.

$$
y_{i j s}\left(\frac{\rho_{j s}-\mu_{i}^{\rho}}{\sigma_{i}^{\rho}}-\Gamma_{\Theta_{j s}^{\rho}}\right) \geqslant 0 \forall s \in \Omega, \forall j \in J_{s}, \forall i \in I
$$

Eq.(12) is used for ensuring the minimum demand satisfaction level met under each scenario considered. Eq. (13) ensures that total amount of allocated (satisfied) bandwidth portion of each end-user can not be more than their requests under all scenarios. The constraint set (14) guarantees that total amount of satisfied bandwidth demand of a customer has to be equal to sum of allocated bandwidth portions of that demand into BPs.

$$
\begin{aligned}
& \sum_{j \in J_{s}} y_{j s} d_{j s}-\pi \sum_{j \in J_{s}} d_{j s} \geqslant 0, \forall s \in \Omega \\
& \sum_{i \in I} y_{i j s} \leqslant 1, \forall s \in \Omega, \forall j \in J_{s} \\
& y_{j s}=\sum_{i \in I} y_{i j s}, \forall s \in \Omega, \forall j \in J_{s} \\
& \beta_{i}, y_{i j s}, y_{j s} \geqslant 0, \forall s \in \Omega, \forall j \in J_{s}, \forall i \in I
\end{aligned}
$$




\section{Computational Experiments}

For computational study, three different types of problem setting with changing number of BPs $(|I|)$, end-users $(|J|)$ and scenarios $(|S|)$ are chosen. Five different and independent problem instances are generated for each type of problem setting as in 52 . For example, problem set I15J50S10 in Table 2 indicates a problem type in which there exists 15 BPs, 50 end-users at market and there exist 10 possible future bandwidth demand scenarios that may occur.

All of the generated problem sets are tested by using different $w_{1}, w_{2}, w_{3}$ values. Changing values of $\left(w_{1}, w_{2}, w_{3}\right)$ are chosen from set $\{(1,0,0),(0,1,0),(0,0,1)\}$ in order to find the most pessimistic, the most expected and the most optimistic values of net profit.

Each end-user's demand $d_{j s}$ is generated from normal distribution. However, to prevent negative bandwidth demand generation from normal distribution, truncation procedure is applied. The realization probabilities of each scenario are generated from uniform distribution between $[0,1]$ afterwards these probabilities are normalized.

The capacity acquisition and allocation problem of BB that is modeled in previous section provides so-called here-and-now solution, and the entire FSLP model named as recourse problem (RP) model. The optimal objective function value of RP (Eq.(6)) is denoted as $\widetilde{z}^{R P}$ and it represents optimal fuzzy expected net profit $(\widetilde{E N P})$. The most optimistic value $\left(E N P^{U}\right)$, the most expected $\left(E N P^{M}\right)$ and the most pessimistic $\left(E N P^{L}\right)$ values of $\widetilde{E N P}$ can be calculated by setting $\left(w_{1}, w_{2}, w_{3}\right)$ parameters appropriately. In addition, objective function components of FSLP such as fuzzy expected total revenue $(\widetilde{E T R})$, fuzzy capacity leasing cost $(\widetilde{C L C})$ and fuzzy expected opportunity cost $(\widetilde{E R C})$ occurring due to unmeet bandwidth requests can be calculated in same manner. It should be noted that below equality among these quantities has to be hold.

$$
\widetilde{E N P}=\widetilde{E T R} \ominus \widetilde{C L C} \ominus \widetilde{E R C}
$$

Where $\ominus$ indicates subtraction operation between 3- tuples which is defined as in Eq.(17).

$$
\begin{aligned}
& E N P^{L}=E T R^{L}-C L C^{L}-E R C^{L} \\
& E N P^{M}=E T R^{M}-C L C^{M}-E R C^{M} \\
& E N P^{U}=E T R^{U}-C L C^{U}-E R C^{U}
\end{aligned}
$$

Table 2 summaries the averaged results under each problem setting for RP model. The increasing number of end-users $|J|$ in the market leads to sharp increasing in fuzzy ENP value under every problem setting.

Table 2. Optimal fuzzy ENP, ETR, CLC and ERC values for RP problem.

\begin{tabular}{lccc}
\hline & \multicolumn{3}{c}{ ENP } \\
Problem Set & $\left(w_{1}, w_{2}, w_{3}\right)$ & $\left(\begin{array}{c}\left.w_{1}, w_{2}, w_{3}\right) \\
(0,1,0)\end{array}\right.$ & $\begin{array}{c}\left(w_{1}, w_{2}, w_{3}\right) \\
(0,0,1)\end{array}$ \\
\hline I15J50S10 & 7513.08 & 7705.28 & 7911.53 \\
I30J100S50 & 15517.57 & 16044.32 & 16569.62 \\
I50J100S100 & 16473.62 & 17226.41 & 17987.00 \\
\hline & & ETR & \\
Problem Set & $(1,0,0)$ & $\left(w_{1}, w_{2}, w_{3}\right)$ & $\left(w_{1}, w_{2}, w_{3}\right)$ \\
I15J50S10 & 12070.14 & 13435.21 & $(0,0,1)$ \\
I30J100S50 & 24108.80 & 26806.95 & 14772.72 \\
I50J100S100 & 24154.19 & 26863.02 & 29491.57 \\
\hline & & CLC & \\
& $\left(w_{1}, w_{2}, w_{3}\right)$ & $\left(w_{1}, w_{2}, w_{3}\right)$ & $\left(w_{1}, w_{2}, w_{3}\right)$ \\
Problem Set & $(1,0,0)$ & $(0,1,0)$ & $(0,0,1)$ \\
I15J50S10 & 4533.05 & 5702.69 & 6825.55 \\
I30J100S50 & 8558.61 & 10719.46 & 12865.38 \\
I50J100S100 & 7656.27 & 9604.02 & 11537.30 \\
\hline
\end{tabular}

When the most optimistic and pessimistic values of ENP is compared, $5.30 \%, 6.78 \%$ and $9.19 \%$ differences are observed for problems sets I15J50S10, I30J100S50 and I50J100S100, respectively. Therefore, it can be concluded that increasing uncertainty via number of scenarios, end-users and backbones causes larger gaps between optimistic and pessimistic values of ENP. On the other hand, the percentage gap between optimistic and pessimistic values remain unchanged for ETR and CLC statistics namely the gaps stay around $22.3 \%$ and $50 \%$ for ETR and CLC, respectively. The gap for ERC 
increases from $48 \%$ to $72 \%$ when problem type changed from I15J50S10 to I50J100S100.

Table 3 presents averaged secondary performance statistics such as fuzzy total capacity bought (TCB), fuzzy capacity loss (CL), fuzzy expected capacity utilization percentage (ECUP) and fuzzy number of selected backbone providers (NSS) for RP model. For a given value of $w$, statistics ECUP and CL can be evaluated as follows:

$$
\begin{aligned}
& \operatorname{ECUP}(w)=\frac{\sum_{s \in \Omega} \sum_{j \in J_{s}} p_{s} y(w)_{j s} d_{j s}}{\sum_{i \in I} \beta(w)_{i}} \\
& C L(w)=\sum_{i \in I} \alpha_{i} \beta(w)_{i}
\end{aligned}
$$

The natural consequence of increasing number of end-users in the market is the more bandwidth acquisition from BPs for BB. CL statistic and TCB are positively correlated which means that higher amount of capacity purchase causes increase in the amount of total capacity loss.

Table 3. Optimal fuzzy TCB, CL, ECUP and NSS values for

\begin{tabular}{|c|c|c|c|}
\hline Problem Set & $\begin{array}{c}\left(w_{1}, w_{2}, w_{3}\right) \\
(1,0,0)\end{array}$ & $\begin{array}{c}\text { TCB } \\
\left(w_{1}, w_{2}, w_{3}\right) \\
(0,1,0)\end{array}$ & $\begin{array}{c}\left(w_{1}, w_{2}, w_{3}\right) \\
(0,0,1)\end{array}$ \\
\hline I15J50S 10 & 489.90 & 489.86 & 487.87 \\
\hline I30J100S50 & 982.48 & 981.71 & 980.63 \\
\hline \multirow[t]{2}{*}{ I50J100S100 } & 981.47 & 980.87 & 979.76 \\
\hline & $\begin{array}{c}\left(w_{1}, w_{2}, w_{3}\right) \\
(1,0,0)\end{array}$ & $\begin{array}{c}\mathbf{C L} \\
\left(w_{1}, w_{2}, w_{3}\right) \\
(0,1,0)\end{array}$ & $\begin{array}{c}\left(w_{1}, w_{2}, w_{3}\right) \\
(0,0,1)\end{array}$ \\
\hline I15J50S10 & 39.44 & 39.48 & 39.30 \\
\hline I30J100S50 & 82.18 & 82.26 & 82.29 \\
\hline \multirow[t]{2}{*}{ I50J100S 100} & 80.22 & 80.34 & 80.34 \\
\hline & $\begin{array}{c}\left(w_{1}, w_{2}, w_{3}\right) \\
(1,0,0)\end{array}$ & $\begin{array}{c}\text { ECUP } \\
\left(w_{1}, w_{2}, w_{3}\right) \\
(0,1,0)\end{array}$ & $\begin{array}{c}\left(w_{1}, w_{2}, w_{3}\right) \\
(0,0,1)\end{array}$ \\
\hline I15J50S10 & 91.11 & 91.11 & 91.24 \\
\hline I30J100S50 & 90.93 & 90.89 & 90.85 \\
\hline I50J100S100 & 91.17 & 91.13 & 91.13 \\
\hline Problem Set & $\begin{array}{c}\left(w_{1}, w_{2}, w_{3}\right) \\
(1,0,0)\end{array}$ & $\begin{array}{c}\text { NSS } \\
\left(w_{1}, w_{2}, w_{3}\right) \\
(0,1,0)\end{array}$ & $\begin{array}{c}\left(w_{1}, w_{2}, w_{3}\right) \\
(0,0,1)\end{array}$ \\
\hline I15J50S10 & 6.8 & 6.8 & 6.8 \\
\hline I30J100S50 & 13.4 & 13.2 & 13.4 \\
\hline I50J100S100 & 12.8 & 13 & 13 \\
\hline
\end{tabular}
RP problem.

When the entire problem instances investigated, it can be seen that the acquired capacity is utilized around $90 \%$ (ECUP statistic). Interestingly, there is no obvious relationship between the changing value of $w$ and CL, NSS and ECUP measures.

Another important statistics needed for decision makers of BP is the expected demand fulfillment ratio (EDFR), which provides information about the ratio of the satisfied bandwidth requests to total bandwidth requests of end-users. The higher value is needed to establish long term relationship with clients. Low EDFR may cause to diminishment in ENP values due to the decreasing capacity requests. Figure 6 depicts the averaged fuzzy EDFR for each problem type. EDFR statistic fluctuates between $98.6 \%$ and $99.5 \%$ and it is effected by $w$ values. Moreover, it is observed that EDFR is relatively higher for the test instances in which total amount of bandwidth request is excessive.

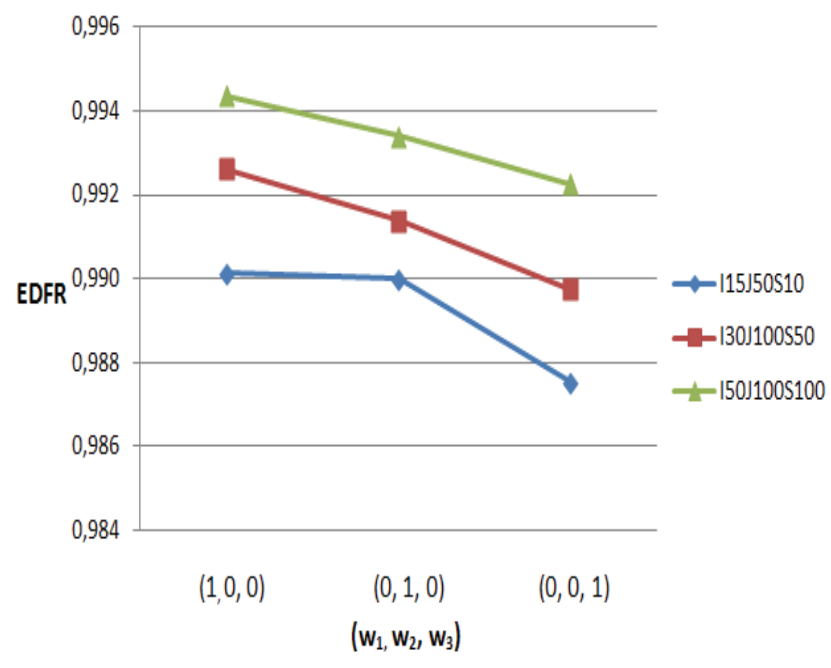

Fig. 6. The effect of altering values of $w$ on EDFR statistic for RP model.

\subsection{Fuzzy Expected Value of Perfect Information $\widetilde{(E V P I})$ and Fuzzy Value of Stochastic Solution $(\widetilde{V S S})$}

In order to test applicability of proposed model, two new models which are called as WS and EEV have to set up and solved. WS model defines wait-andsee solution of the model and its optimal objective function value is $\widetilde{z}^{W S}$. The expected value of perfect 
information (EVPI) measures the maximum amount that a decision maker is willing to pay in order to know the value of a random variable before making his/her decision ${ }^{53}$. In this paper, fuzzy EVPI is calculated as following,

$$
\widetilde{\mathrm{EVPI}}=\widetilde{z}^{W S} \ominus \widetilde{z}^{R P}
$$

$\ominus$ does not indicate fuzzy subracation operation, due to the fact that decision maker of BB may choose same $w$ values while solving both RP and WS models ${ }^{52}$. The suggested fuzzy EVPI algorithm is presented in Figure 7.

\begin{tabular}{ll}
\hline for & $s=1, \ldots,|\Omega|$ \\
& Fix $\left(w_{1}, w_{2}, w_{3}\right)=(1,0,0)$ \\
& Solve Eqs. $(5-15)$ \\
& Save $z_{s}^{L}$ \\
& Fix $\left(w_{1}, w_{2}, w_{3}\right)=(0,1,0)$ \\
& Solve Eqs. $(5-15)$ \\
& Save $z_{s}^{M}$ \\
& Fix $\left(w_{1}, w_{2}, w_{3}\right)=(0,0,1)$ \\
& Solve Eqs. $(5-15)$ \\
& Save $z_{s}^{U}$ \\
& \\
end for & $z_{L}^{W S} \leftarrow \sum_{s=1}^{|\Omega|} p_{s} z_{s}^{L}$ \\
Calculate and Assign & $z_{M}^{W S} \leftarrow \sum_{s=1}^{|\Omega|} p_{s} z_{s}^{M}$ \\
& $z_{U}^{W S} \leftarrow \sum_{S=1}^{|\Omega|} p_{s} z_{s}^{U}$ \\
& $\widetilde{z}^{W S}=\left(z_{L}^{W S}, z_{M}^{W S}, z_{U}^{W S}\right)$ \\
& \\
Calculate and Assign & $\mathrm{EVPI}^{L} \leftarrow z_{L}^{W S}-z_{L}^{R P}$ \\
& $\mathrm{EVPI}^{M} \leftarrow z_{M}^{W S}-z_{M}^{R P}$ \\
& $\mathrm{EVPI}^{U} \leftarrow z_{U}^{W S}-z_{U}^{R P}$ \\
\hline
\end{tabular}

Fig. 7. The proposed $\widetilde{\text { EVPI }}$ algorithm.

Just solving RP and WS models do not provide too much information to decision makers. Thus, BB has to measure the increase in fuzzy ENP obtained by solving the FSLP instead of the expected value problem $(\mathrm{EV})$ that is attained by replacing all random variables by their expected values ${ }^{54}$. EEV is calculated by the expected objective value obtained while using the optimal solution of the deterministic problem EV. The Value of Stochastic Solution (VSS) measure combines RP and EEV and compares the here-and-now and expected value approaches ${ }^{53}$. The simpler version of FSLP is formulated between Eq.(20) and Eq.(28). EV model essentially tries to maximize fuzzy profit rather than expectation of it by not taking into account randomness arising in market and infrastructure. In EV model, none of the parameters depends on future scenarios that may occur.

$$
\operatorname{Maximize}\left\{\begin{array}{l}
w_{1}\left(\sum_{j \in J} \vartheta_{j}^{L} y_{j}-\sum_{i \in I} \beta_{i} c_{i}^{L}-\sum_{j \in J} v_{j}^{L}\left(1-y_{j}\right)\right)+ \\
w_{2}\left(\sum_{j \in J} \vartheta_{j}^{M} y_{j}-\sum_{i \in I} \beta_{i} c_{i}^{M}-\sum_{j \in J} v_{j}^{M}\left(1-y_{j}\right)\right)+ \\
w_{3}\left(\sum_{j \in J} \vartheta_{j}^{U} y_{j}-\sum_{i \in I} \beta_{i} c_{i}^{U}-\sum_{j \in J} v_{j}^{U}\left(1-y_{j}\right)\right)
\end{array}\right.
$$

Subject to: $\sum_{j \in J} y_{i j} d_{j} \leqslant\left(1-\alpha_{i}\right) \beta_{i}, \forall i \in I$

$$
\begin{aligned}
& \beta_{i} \leqslant U_{i}, \forall i \in I \\
& y_{i j}\left(\frac{\delta_{j}-\mu_{i}^{\delta}}{\sigma_{i}^{\delta}}-\Gamma_{\Theta_{j}^{\delta}}\right) \geqslant 0 \forall j \\
& y_{i j}\left(\frac{\rho_{j}-\mu_{i}^{\rho}}{\sigma_{i}^{\rho}}-\Gamma_{\Theta_{j}^{\rho}}\right) \geqslant 0 \forall j \\
& \sum_{j \in J} y_{j} d_{j}-\pi \sum_{j \in J} d_{j} \geqslant 0, \\
& \sum_{i \in I} y_{i j} \leqslant 1, \forall j \in J \\
& y_{j}=\sum_{i \in I} y_{i j}, \forall j \in J \\
& \beta_{i}, y_{i j}, y_{j} \geqslant 0, \forall j \in J, \forall i \in I
\end{aligned}
$$$$
y_{i j}\left(\frac{\delta_{j}-\mu_{i}^{\delta}}{\sigma_{i}^{\delta}}-\Gamma_{\Theta_{j}^{\delta}}\right) \geqslant 0 \forall j \in J, \forall i \in I(23)
$$$$
y_{i j}\left(\frac{\rho_{j}-\mu_{i}^{\rho}}{\sigma_{i}^{\rho}}-\Gamma_{\Theta_{j}^{\rho}}\right) \geqslant 0 \forall j \in J, \forall i \in I(24)
$$

The bandwidth acquisition amounts from each BP $\left(\beta_{i}^{E V}\right)$ at optimal solution of EV under different $w$ values are used as input for EEV model rather than decision variables. The optimal fuzzy objective value of EEV model is denoted as $\widetilde{z}^{E E V}$. The difference between $\widetilde{z}^{R P}$ and $\widetilde{z}^{E E V}$ is called as value of fuzzy stochastic solution(VSS) which is also a fuzzy number and calculated as $\widetilde{V S S}=\widetilde{z}^{R P} \ominus \widetilde{z}^{E E V}$. Eq. 29 shows detailed calculation for $\widetilde{V S S}$. Here, again $\ominus$ does not indicate fuzzy subtraction operation, due to the fact that decision maker of BB may choose same $w$ values while solving both RP and EEV models 52. Fuzzy VSS measures the cost of ignoring uncertainty while making a decision ${ }^{1}$. The proposed fuzzy VSS algorithm is presented in Figure 8.

$$
\begin{aligned}
\widetilde{V S S}= & \left(V S S^{L}, V S S^{M}, V S S^{U}\right)= \\
& \left(z_{L}^{R P}-z_{L}^{E E V}, z_{M}^{R P}-z_{M}^{E E V}, z_{U}^{R P}-z_{U}^{E E V}\right)(29)
\end{aligned}
$$

Table 4 summarizes results gathered from running fuzzy VSS and fuzzy EVPI algorithms for each problem set on RP, EEV and WS models. All of the 
results are presented in TFN format. Problem set I15J50S10_3 does not have a feasible solution when optimal solution of $\mathrm{EV}\left(\beta_{i, L}^{E V}, \beta_{i, M}^{E V}, \beta_{i, U}^{E V}\right)$ is used in RP model, which is indicated via $* * * *$. This situation occurs due to the fact that optimal amount of bandwidth acquired in EV model may not satisfy minimum required demand fulfillment ratio in RP problem (see Eq.(12)). As problem size gets larger, $\widetilde{V S S}$ value increases due to the increasing uncertainty added by increasing number of scenarios, end-users and BPs. In addition, $\widetilde{V S S}$ values always greater than zero $(\succ 0)$ which implies the fact that solving RP model rather than EEV model leads to more profit in fuzzy sense. In other words, ignoring uncertainty in the bandwidth demand and treating BB's problem as deterministic model result in profit loss. It should be also noted that for all of the test instances solved optimally $\widehat{V S S} \succ \widehat{E V P I}$ inequality holds.

Table 4. Fuzzy VSS and EVPI values for each problem set.

\begin{tabular}{lcc}
\hline \hline Problem Set & $\widehat{\text { VSS }}$ & $\widehat{\text { EVPI }}$ \\
\hline I15J50S10_0 & $(134.37,168.39,208.35)$ & $(55.72,59.61,63.32)$ \\
I15J50S10_1 & $(512.87,663.86,814.63)$ & $(164.48,174.91,186.66)$ \\
I15J50S10_2 & $((951.67,1068.25,1183.52)$ & $(100.94,118.40,139.06)$ \\
I15J50S10_3 & $(* * *, * * *, * * *)$ & $(520.52,607.66,692.04)$ \\
I15J50S10_4 & $(1499.31,1673.50,1850.60)$ & $(207.18,253.38,294.84)$ \\
\hline Average & $(776.96,903.76,1033.48)$ & $(209.77,242.79,275.18)$ \\
\hline Problem Set & $\widehat{V S S}$ & $\widehat{E V P I}$ \\
\hline I30J100S50_0 & $(1394.51,1755.50,2116.63)$ & $(276.13,327.98,371.44)$ \\
I30J100S50_1 & $(1221.35,1542.25,1854.57)$ & $(247.08,295.03,344.69)$ \\
I30J100S50_2 & $(927.40,1190.52,1452.70)$ & $(420.09,492.81,556.95)$ \\
I30J100S50_3 & $(2431.03,3051.87,3690.88)$ & $(646.67,763.28,872.95)$ \\
I30J100S50_4 & $(1827.09,2297.66,2777.53)$ & $(560.96,674.12,786.42)$ \\
\hline Average & $(1560.28,1967.56,2378.46)$ & $(430.19,510.64,586.49)$ \\
\hline Problem Set & $\widehat{\text { VSS }}$ & $\widehat{\text { EVPI }}$ \\
\hline I50J100S100_00 & $(1728.44,2150.82,2599.03)$ & $(357.88,433.65,506.07)$ \\
I50J100S100_1 & $(2243.64,2834.07,3421.93)$ & $(469.44,559.06,643.20)$ \\
I50J100S100_2 & $(1817.50,2306.58,2790.38)$ & $(509.34,602.77,694.18)$ \\
I50J100S100_3 & $(1190.82,1504.82,1799.91)$ & $(325.80,395.06,460.78)$ \\
I50J100S100_4 & $(1996.56,2496.65,2997.80)$ & $(217.10,253.60,293.26)$ \\
\hline Average & $(1795.39,2258.59,2721.81)$ & $(375.91,448.83,519.50)$ \\
\hline \hline
\end{tabular}

By using data presented in Table 4, the improvement ratios attained by RP model over EEV model can be calculated. In the same manner, the improvement ratios that can be achieved by knowing the future bandwidth demands and prices may also be calculated from presented data. Besides, decision maker of BB may set up a lower threshold ratio based on his experiences to determine which model to solve (RP or EEV).

\begin{tabular}{|c|c|}
\hline for & $\begin{array}{l}j=1, \ldots,|J| \\
\text { Calculate and Assign } \\
\widetilde{\vartheta}_{j} \leftarrow \sum_{s \in \Omega}^{\oplus} p_{s} \otimes \widetilde{\vartheta}_{j s} \\
\widetilde{v}_{j} \leftarrow \sum_{s \in \Omega} p_{s} \otimes \widetilde{v}_{j s} \\
d_{j} \leftarrow \sum_{s \in \Omega} p_{s} d_{j s} \\
\delta_{j} \leftarrow \sum_{s \in \Omega} p_{s} \delta_{j s} \\
\rho_{j} \leftarrow \sum_{s \in \Omega} p_{s} \rho_{j s} \\
\Theta_{j}^{\delta} \leftarrow \sum_{s \in \Omega} p_{s} \Theta_{j s}^{\delta} \\
\Theta_{j}^{\rho} \leftarrow \sum_{s \in \Omega} p_{s} \Theta_{j s}^{\rho}\end{array}$ \\
\hline end for & \\
\hline Fix & $\left(w_{1}, w_{2}, w_{3}\right)=(1,0,0)$ in Eq. $(20)$ \\
\hline Solve & Eqs. $(20-28)$ \\
\hline Save & $\beta_{i, L}^{E V}$ \\
\hline Fix & $\left(w_{1}, w_{2}, w_{3}\right)=(1,0,0)$ in Eq. $(5)$ \\
\hline Assign and Fix & $\beta_{i} \leftarrow \beta_{i, L}^{E V}$ in Eqs. $(6,7,15)$ \\
\hline Solve & $\begin{array}{l}\text { RP problem in Eq.(5-15) } \\
\text { Optimal solution } z_{L}^{E E V}\end{array}$ \\
\hline Fix & $\left(w_{1}, w_{2}, w_{3}\right)=(0,1,0)$ in Eq. $(20)$ \\
\hline Solve & Eqs.(20-28) \\
\hline Save & $\beta_{i, M}^{E V}$ \\
\hline Fix & $\left(w_{1}, w_{2}, w_{3}\right)=(0,1,0)$ in Eq. (5) \\
\hline Assign and Fix & $\beta_{i} \leftarrow \beta_{i, M}^{E V}$ in Eqs. $(6,7,15)$ \\
\hline Solve & $\begin{array}{l}\text { RP problem in Eqs.(5-15) } \\
\text { Optimal solution } z_{M}^{E E V}\end{array}$ \\
\hline Fix & $\left(w_{1}, w_{2}, w_{3}\right)=(0,0,1)$ in Eq. $(20)$ \\
\hline Solve & Eqs.(20-28) \\
\hline Save & $\beta_{i, U}^{E V}$ \\
\hline Fix & $\left(w_{1}, w_{2}, w_{3}\right)=(0,0,1)$ in Eq. $(5)$ \\
\hline Assign and Fix & $\beta_{i} \leftarrow \beta_{i U}^{E V}$ in Eqs. $(6,7,15)$ \\
\hline Solve & $\begin{array}{l}\text { RP problem in Eq.(5-15) } \\
\text { Optimal solution } z_{U}^{E E V}\end{array}$ \\
\hline Calculate and Assign & $\begin{array}{l}\mathrm{VSS}^{L} \leftarrow z_{L}^{R P}-z_{L}^{E E V} \\
\mathrm{VSS}^{M} \leftarrow z_{M}^{R P}-z_{M}^{E E V} \\
\mathrm{VSS}^{U} \leftarrow z_{U}^{R P}-z_{U}^{E E V}\end{array}$ \\
\hline
\end{tabular}

Fig. 8. The proposed $\widetilde{\text { VSS }}$ algorithm.

For mentioned reasons two new statistics are defined as follows:

$$
\begin{aligned}
& \widetilde{\zeta}=\widetilde{V S S} \odot \widetilde{z}^{E E V}=\left(\frac{V S S^{L}}{z_{L}^{E E V}}, \frac{V S S^{M}}{z_{M}^{E E V}}, \frac{V S S^{U}}{z_{U}^{E E V}}\right) \\
& \widetilde{\xi}=\widetilde{E V P I} \odot \widetilde{z}^{R P}=\left(\frac{E V P I^{L}}{z_{L}^{R P}}, \frac{E V P I^{M}}{z_{M}^{R P}}, \frac{E V P I^{U}}{z_{U}^{R P}}\right)
\end{aligned}
$$

$\widetilde{\zeta}$ measures the advantage of fuzzy stochastic approach (RP) over deterministic approach (RP), and $\widetilde{\xi}$ measures how much more profit can be obtained 
by knowing the future bandwidth demands, prices and market conditions in advance. Table 5 presents calculated $\widetilde{\zeta}$ and $\widetilde{\xi}$ values.

Table 5. Calculated $\widetilde{\zeta}$ and $\widetilde{\xi}$ values for each problem instances.

\begin{tabular}{lcc}
\hline \hline Problem Set & $\widetilde{\zeta}$ & $\tilde{\xi}$ \\
\hline I15J50S10_0 & $(0.018,0.022,0.026)$ & $(0.007,0.008,0.008)$ \\
I15J50S10_1 & $(0.076,0.098,0.119)$ & $(0.023,0.023,0.024)$ \\
I15J50S10_2 & $(0.142,0.157,0.172)$ & $(0.013,0.015,0.017)$ \\
I15J50S10_3 & $(* * * *, * * * *, * * *)$ & $(0.069,0.078,0.087)$ \\
I15J50S10_4 & $(0.253,0.284,0.315)$ & $(0.028,0.033,0.038)$ \\
Average & $(0.115,0.133,0.150)$ & $(0.028,0.032,0.035)$ \\
\hline Problem Set & $\widetilde{\zeta}$ & $\xi$ \\
\hline I30J100S50_0 & $(0.089,0.109,0.128)$ & $(0.016,0.018,0.020)$ \\
I30J100S50_1 & $(0.087,0.108,0.129)$ & $(0.016,0.019,0.021)$ \\
I30J100S50_2 & $(0.065,0.082,0.098)$ & $(0.027,0.031,0.034)$ \\
I30J100S50_3 & $(0.201,0.259,0.323)$ & $(0.045,0.051,0.058)$ \\
I30J100S50_4 & $(0.134,0.168,0.203)$ & $(0.036,0.042,0.048)$ \\
Average & $(0.112,0.140,0.168)$ & $(0.028,0.032,0.035)$ \\
\hline Problem Set & $\widetilde{\zeta}$ & $\widetilde{\xi}$ \\
\hline I50J100S100_0 & $(0.125,0.155,0.186)$ & $(0.023,0.027,0.030)$ \\
I50J100S100_1 & $(0.160,0.200,0.239)$ & $(0.029,0.033,0.036)$ \\
I50J100S100_2 & $(0.123,0.153,0.181)$ & $(0.031,0.035,0.038)$ \\
I50J100S100_3 & $(0.076,0.092,0.107)$ & $(0.019,0.022,0.025)$ \\
I50J100S100_4 & $(0.133,0.163,0.191)$ & $(0.013,0.014,0.016)$ \\
Average & $(0.122,0.151,0.178)$ & $(0.023,0.026,0.029)$ \\
\hline \hline
\end{tabular}

One of the most important results is the statistic $\widetilde{\zeta}$ is always positive and bigger than the $\widetilde{\xi}$ value, which indicates superiority of RP (fuzzy stochastic) modeling over EEV. The close investigation of Table 5 reveals that averages of mentioned statistics gets larger as increasing problem size in other words increasing uncertainty in both market prices and demand is correlated with $\widetilde{\zeta}$ value.

\subsection{Sensitivity Analysis}

Another important issue for decision makers is to test the stability of solution methodology under dynamic settings such as changing fuzzy revenues and costs. In this direction, the largest problem set I50J100S100 is tested under varying fuzzy objective parameters.

As a first part of the sensitivity analysis, all of the fuzzy parameters in objective function of RP model such as $\left(\widetilde{\vartheta}_{j s}, \widetilde{v}_{j s}, \widetilde{c}_{i}\right)$ are increased and decreased $20 \%$ from their initial values under every demand scenario. For example, the increments and decrements operations for fuzzy unit revenues under scenario $s$ are performed as follows:

$$
\widetilde{\vartheta}_{j s} \longleftarrow\left(1.2 \vartheta_{j s}^{L}, 1.2 \vartheta_{j s}^{M}, 1.2 \vartheta_{j s}^{U}\right)
$$

$$
\widetilde{\vartheta}_{j s} \longleftarrow\left(0.8 \vartheta_{j s}^{L}, 0.8 \vartheta_{j s}^{M}, 0.8 \vartheta_{j s}^{U}\right)
$$

Table 6 shows effect of $\pm 20 \%$ change in fuzzy objective parameters on $\widetilde{V S S}, \widetilde{E V P I}, \widetilde{\zeta}, \widetilde{\xi}$ values. Even though, increase in fuzzy parameters leads to increment for $\widetilde{V S S}$ and $\widetilde{E V P I}$, it does not effect $\widetilde{\zeta}$ and $\widetilde{\xi}$.

Table 6 . The effect of $\pm 20 \%$ change in fuzzy objective parameters on $\widetilde{V S S}, \widetilde{E V P I}, \widetilde{\zeta}, \widetilde{\xi}$ values.

\begin{tabular}{lcc}
\hline \hline Test scenario & $\widetilde{V S S}$ & $\widetilde{\zeta}$ \\
\hline 20\% increase & $(2154.47,2710.30,3266.17)$ & $(0.122,0.151,0.178)$ \\
Original case & $(1795.39,2258.59,2721.81)$ & $(0.122,0.151,0.178)$ \\
20\% decrease & $(1436.31,1806.87,2177.45)$ & $(0.122,0.151,0.178)$ \\
\hline Test scenario & $\widetilde{E V P I}$ & $\widetilde{\xi}$ \\
\hline 20\% increase & $(451.10,550.00,623.40)$ & $(0.023,0.027,0.029)$ \\
Original case & $(375.91,448.83,519.50)$ & $(0.023,0.026,0.029)$ \\
20\% decrease & $(300.73,359.06,415.60)$ & $(0.023,0.026,0.029)$ \\
\hline \hline
\end{tabular}

In the second part of sensitivity analysis on fuzzy objective parameters, fuzzy unit revenues are altered between $\left(\vartheta_{j s}\right) \pm 10 \%$ from their original values while keeping the other parameters unchanged. RP, WS and EEV models are run under these new settings and main performance indicators $(\widetilde{\zeta}$ and $\widetilde{\xi})$ are evaluated and compared to original cases. Figure 9 and 10 depict results of sensitivity runs on revenue coefficients.

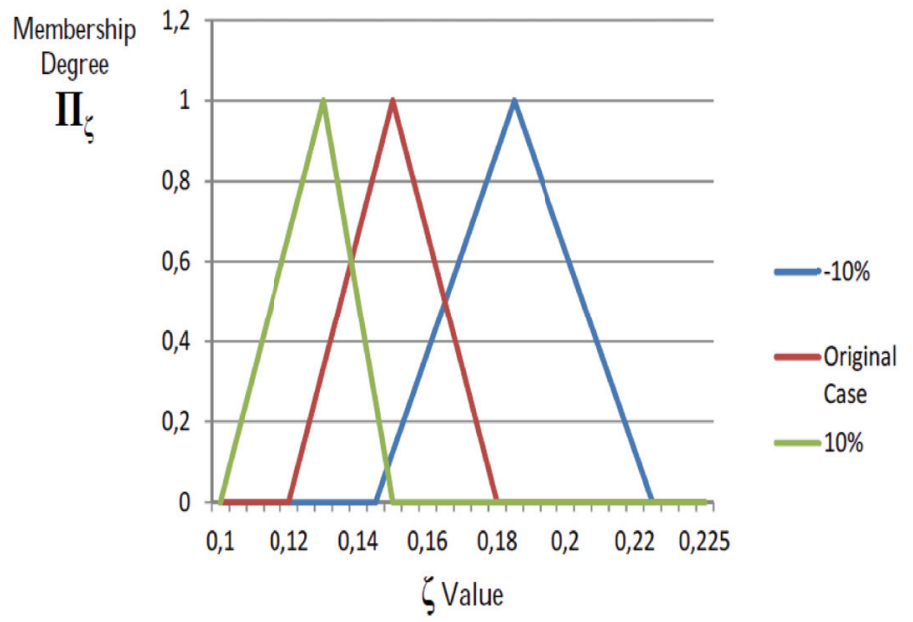

Fig. 9. Effect of change in fuzzy unit revenues by $\pm 10 \%$ on $\widetilde{\zeta}$. 
The increasing revenues lead to decrease in $\widetilde{\zeta}$ values proving that randomness should be taken into account due to the fact that very high competition in telecom market and limited profit margins. As a last part of sensitivity analysis, the fuzzy unit cost namely leasing and opportunity costs $\left(v_{j s}, c_{i}\right)$ are changed between $\pm 10 \%$ from their original settings simultaneously in order to conduct sensitivity analysis on objective parameters. Figure 11 and 12 depict results of sensitivity runs on cost coefficients.

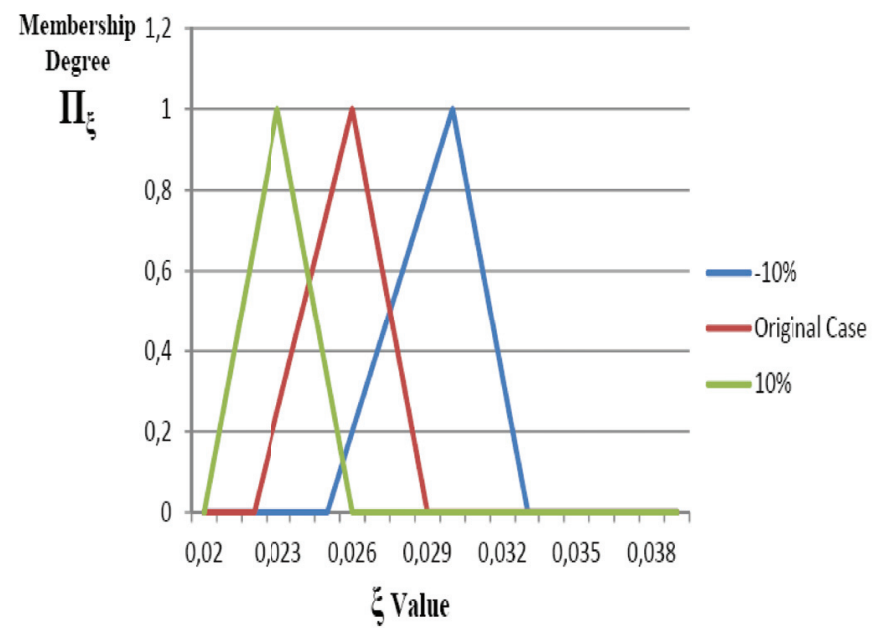

Fig. 10. Effect of change in fuzzy unit revenues by $\pm 10 \%$ on $\widetilde{\xi}$.

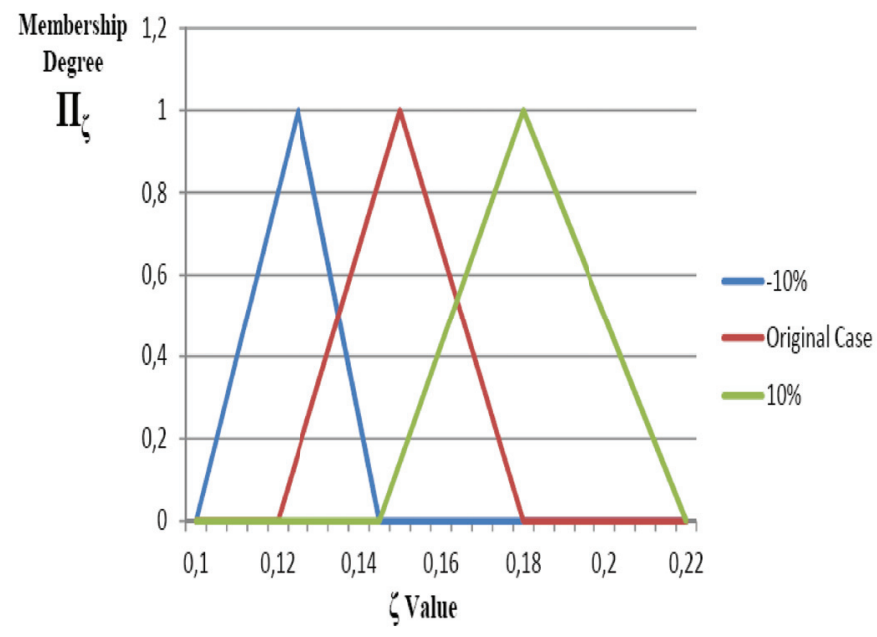

Fig. 11. Effect of change in fuzzy unit costs by $\pm 10 \%$ on $\widetilde{\zeta}$.
The rising costs cause $\widetilde{\zeta}$ to rise as well, which verifies that RP model better to be chosen and used by $\mathrm{BB}$ under pessimistic market conditions.

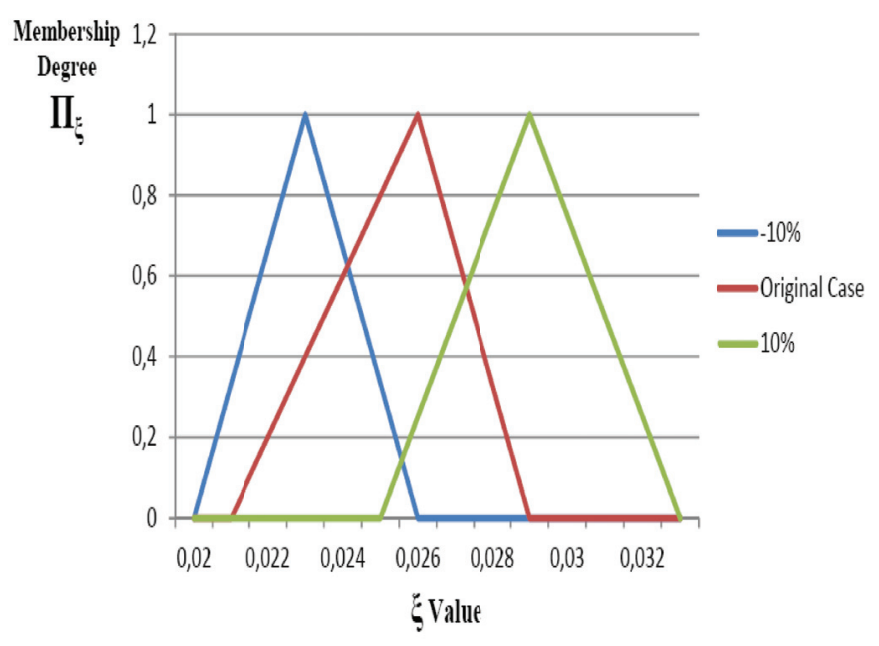

Fig. 12. Effect of change in fuzzy unit costs by $\pm 10 \%$ on $\widetilde{\xi}$.

\section{Conclusions}

In this research, an optimization problem of BB is analyzed when acquiring and allocating bandwidth from a market in which demands of end-users, prices and QoS levels are not known in advance. The main contribution of the paper is to develop an efficient solution algorithm to overcome computational issues as well as uncertainties in the non-deterministic nature of the telecommunication network environment. In order to handle randomness and vagueness, an integration of stochastic and fuzzy linear programming techniques is proposed.

Extensive computational study on randomly generated test instances showed that proposed methodology provides more than $10 \%$ more profit even in worst case scenarios. We analyze the effect of fuzziness in managerial point of view. We show that randomness should be taken into account due to very high competition in telecom market and limited profit margins.

It is concluded that increasing problem size makes suggested approach more compelling than deterministic methodology for BB's decision makers. Solving RP model rather than EEV model leads 
to more profit in fuzzy sense. In other words, ignoring uncertainty in the bandwidth demand and treating BB's problem as deterministic model result in profit loss. Moreover, the RP model is better to be chosen and used by BB under pessimistic market conditions. Finally, carried out sensitivity analysis on fuzzy objective parameters demonstrated the robustness of procedure.

As a further study, it would be worthwhile to expand investigated two-stage model into multi-period (multi-stage) model. In addition, an interesting future study would be to study fuzzy multi-objective optimization problem of BP rather than BB in which fuzzy expected profit maximization as well as fuzzy expected capacity loss minimization objectives are optimized simultaneously. Even though most of the time it is sufficient to model imprecise parameters by triangular fuzzy number, improving proposed methodology that is capable of handling general fuzzy number would make solution methodology more applicable, which would be a theoretical contribution.

\section{Acknowledgements}

Preliminary findings of this article was presented in ISCSE 2013 Proceedings book with title the "A Fuzzy Capacity Acquisition and Allocation Model for Bandwidth Brokers under Uncertainty ${ }^{52}$ ".

\section{References}

1. Turan, H. H., June 2012. Profit maximization problem for intermediaries in telecommunications networks under stochastic demand. Ph.D. thesis, Istanbul Technical University, Istanbul, Turkey, in Turkish.

2. Audestad, J. A., Gaivoronski, A. A., Werner, A., Extending the stochastic programming framework for the modeling of several decision makers: pricing and competition in the telecommunication sector. Annals of Operational Research 142 (2006), 19-39 .

3. Turan, H. H., Serarslan, N., Kasap, N., A fuzzy stochastic model for telecommunications bandwidth brokers under probabilistic QoS measures. Applied Mathematical Modelling 38 (1)(2014), 12-27.

4. Çanakoğlu, E., Bilgiç, T., Analysis of a two-stage telecommunication supply chain with technology dependent demand. European Journal of Operational Research 177 (2007), 995-1012.
5. Safari, E., Babakhani, M., Sadjadi, S. J., Shahanaghi, K., Naboureh, K., Determining strategy of pricing for a web service with different QoS levels and reservation level constraint. Applied Mathematical Modelling 39 (2015), 3784-3813.

6. Ozianyi, V. G., Ventura, N., Golovins, E., A novel pricing approach to support QoS in 3G networks. Computer Networks 52 (2008), 1433-1450.

7. Guerrero-Ibanez, A., Contreras-Castillo, J., Barba, A., Reyes, A., A QoS-based dynamic pricing approach for services provisioning in heterogeneous wireless access networks. Pervasive and Mobile Computing 7 (2011), 569-583.

8. Si-feng, Z., Fang, L., Yu-tao, Q., Zheng-yi, C., Jianshe, W., Immune optimization algorithm for solving joint call admission control problem in nextgeneration wireless network. Engineering Applications of Artificial Intelligence 25 (2012), 1395-1402.

9. Safari, E., Babakhani, M., Sadjadi, S. J., Shahanaghi, K., Naboureh, K. Dynamic Pricing of a Web Service in an Advance Selling Environment. Mathematical Problems in Engineering (2015), http://dx.doi.org/10.1155/2015/783149.

10. Kelly, F. P., On tariffs, policing and admission control for multi-service networks. Operations Research Letters 15 (1998), 1-9.

11. Courcoubetis, C., Kelly, F., Weber, R., Measurementbased usage charges in communications networks. Operations Research 48 (4) (2000), 535-548.

12. Altmann, J., Chu, K., How to charge for network services - flat rate or usage-based? Computer Networks 36 (2001), 519-531.

13. Gupta, A., Stahl, D. O., Whinston, A. B., 1997. Priority pricing of the integrated services networks. In: McKnight, L. W., Bailey, J. P. (Eds.), Internet Economics, 3rd Edition. The MIT Press.

14. Puspita, F. M., Seman, K., Taib, B. M., Abdullah, I., 2015. Optimization of wireless pricing schemes. In: Proc. of International Conferences on Information Technology and Business (ICITB 2015). p. 89-94.

15. Liu, T., Wang, K., Ku, C., Hsu, Y., QoS-aware resource management for multimedia traffic report systems over LTE-A. Computer Networks (2015), http://dx.doi.org/10.1016/j.comnet.2015.10.021.

16. Malik, A., Qadir, J., Ahmad, B., Yau, K. A., Ullah, U., QoS in IEEE 802.11-based wireless networks: A contemporary review. Journal of Network and Computer Applications 55 (2015), 24-46.

17. Comer, D. E., 2001. Computer Networks and Internets with Internet Applications, 3rd Edition. Prentice Hall, Upper Saddle River, NJ.

18. Gupta, A., Kalyanaraman, S., Zhang, L., Pricing of risk for loss guaranteed intra-domain internet service contracts. Computer Networks 50 (2006), 2787-280

19. Mao, G., A real time loss performance monitoring 
scheme. Computer Communications 28 (2005), 150161.

20. Hoßfeld, T., Binzenhöfer, A., Analysis of Skype VoIP traffic in UMTS: End-to-end QoS and QoE measurements. Computer Networks 52 (2008), 650-666.

21. Karam, M. J., Tobaqi, F. A., Analysis of delay and delay jitter of voice traffic in the Internet Computer Networks 40 (2002), 711-726.

22. Ghosh, S., Razouqi, Q., Schumacher, H. J., Celmins ., A Survey of Recent Advances in Fuzzy Logic in Telecommunications Networks and New Challenges. IEEE Transactions on Fuzzy Systems 6(3) (1998), 443-447.

23. Stavroulakis, P., 2004. Neuro-Fuzzy and FuzzyNeural Applications in Telecommunications. Springer-Verlag Berlin Heidelberg, New York.

24. Sarma, K. K., 2015. Application of Soft Computing Tools in Wireless Communication-A Review. In Sarma, K. K. et al. (eds.), Recent Trends in Intelligent and Emerging Systems. India: Springer.

25. Turan, H. H., Kasap, N., Serarslan, M. N., Single period profit maximization problem for intermediaries in telecommunication networks under stochastic bandwidth demand. Journal of Multiple-Valued Logic and Soft Computing 21 (3-4) (2013), 287-315.

26. Turan, H. H., Kasap, N., Savran, H., A bandwidth sourcing and task allocation model in telecommunications under stochastic QoS guarantees. Applied Soft Computing 24 (2014), 1112-1123.

27. Turan, H. H., Kasap, N., Savran, H., Using heuristic algorithms for capacity leasing and task allocation issues in telecommunication networks under fuzzy quality of service constraints. International Journal of Systems Science 45(3)(2014), 307-324.

28. Uygun, O., Kacamak, H., Kahraman, U. A., An integrated DEMATEL and Fuzzy ANP techniques for evaluation and selection of outsourcing provider for a telecommunication company. Computers \& Industrial Engineering 86 (2015), 137-146.

29. Fu, X., Zhu, Q., Sarkis, J., Evaluating green supplier development programs at a telecommunications systems provider. International Journal of Production Economics 140 (2012), 357-367.

30. Kahraman, C., Engin, O., Kabak, O., Kaya, I., Information systems outsourcing decisions using a group decision-making approach. Engineering Applications of Artificial Intelligence 22 (2009), 832-841.

31. Onut, S., Kara, S. S., Is1k, E., Long term supplier selection using a combined fuzzy MCDM approach: A case study for a telecommunication company. Expert Systems with Applications 36 (2009), 3887-3895.

32. Kabak, O., Ulengin, F., Possibilistic linearprogramming approach for supply chain networking decisions. European Journal Operations Research 3 (209) (2011), 253-264.
33. Mula, J., Polera, R., Garcia-Sabater, J., Material requirement planning with fuzzy constraints and fuzzy coefficients. Fuzzy Sets and Systems 158 (2007), 783 $-793$.

34. Amid, A., Ghodsypour, S. H., O'Brien, C., A weighted additive fuzzy multi objective model for the supplier selection problem under price breaks in a supply chain. International Journal of Production Economics 121 (2009), 323-332.

35. Amid, A., Ghodsypour, S., O’Brien, C., A weighted max-min model for fuzzy multi-objective supplier selection in a supply chain. International Journal of Production Economics 131 (2011), 139 - 145.

36. Ozgen, D., Onut, S., Gulsun, B., Tuzkaya, U. R., Tuzkaya, G., A two-phase possibilistic linear programming methodology for multi-objective supplier evaluation and order allocation problems. Information Sciences 178 (2) (2008), 485-500.

37. Douligeris, C., Computer networks design using hybrid fuzzy expert systems. Soft Computing 5 (2001), 272-286.

38. Wang, J., Fuzzy-Based Routing Scheme in Mobile AD Hoc Networks. Intelligent Automation and Soft Computing 18(3)(2012), 297-305.

39. Ortiz, A. M., Royo, F., Olivares, T., Castillo, J. C., Orozco-Barbosa, L., Marron, P. J., Fuzzy-logic based routing for dense wireless sensor networks. Telecommunication Systems 52 (2013), 2687-2697.

40. Fernandez, M. P., Pedroza, A. C. P., Rezende, J. F., Dynamic QoS Provisioning in DiffServ Domains Using Fuzzy Logic Controllers. Telecommunication Systems 26(1)(2004), 9-32.

41. Mansouri, T., Nabavi, A., Ravasan, A. Z., Ahangarbahan, H., A practical model for ensemble estimation of QoS and QoE in VoIP services via fuzzy inference systems and fuzzy evidence theory. Telecommunication Systems (2015), http://dx.doi.org/10.1007/s11235-015-0041-6.

42. Chen, P., Dong, T., A fuzzy genetic algorithm for QoS multicast routing. Computer Communications 26 (2003), 506-512.

43. Mastorocostas, P., Hilas, C., A computational intelligence-based forecasting system for telecommunications time series. Engineering Applications of Artificial Intelligence 25 (2012), 200-206.

44. Mastorocostas, P. A., Hilas, C. S., Varsamis, D. N., Dova, S. C., Telecommunications call volume forecasting with a block-diagonal recurrent fuzzy neural network. Telecommunication Systems (2015), 1-11.

45. Keropyan, A., Gil-Lafuente, A. M., Customer loyalty programs to sustain consumer fidelity in mobile telecommunication market. Expert Systems with Applications 39 (2012), 11269-11275.

46. Hanafizadeh, P., Mirzazadeh, M., Visualizing market segmentation using self-organizing maps and Fuzzy 
Delphi method - ADSL market of a telecommunication company. Expert Systems with Applications 38 (2011), 198-205.

47. Velmurugan T., Performance based analysis between k-Means and Fuzzy C-Means clustering algorithms for connection oriented telecommunication data. Applied Soft Computing 19 (2014), 134-146.

48. Stula, M, Doko, A., Maras, J., 2011. Country's Internet Spreading Rate Modelling with Fuzzy Cognitive Map. International Journal of Modelling and Simulation 31(4) (2011), 287-295.

49. Azadeh, A., Asadzadeh, S. M., Bukhari, A., Izadbakhsh, R. I., An integrated fuzzy DEA algorithm for efficiency assessment and optimization of wireless communication sectors with ambiguous data. International Journal of Advanced Manufacturing Technology 52 (2011), 805-819.

50. Khademi-Zare, H., Zarei, M., Sadeghieh, A., Owlia, M. S., Ranking the strategic actions of Iran mobile cellular telecommunication using two models of fuzzy QFD. Telecommunications Policy 34 (2010), 747759.

51. Zhang, G., Wu, Y., Remias, M., Lu, J., Formulation of fuzzy linear programming problems as four-objective constrained optimization problems. Applied Mathematics and Computation 139 (2003), 383-399.

52. Turan, H. H., Kasap, N., Delen, D., 2013. A fuzzy capacity acquisition and allocation model for bandwidth broker under uncertainty. In: Proc. of 3rd International Symposium on Computing in Science \& Engineering (ISCSE 2013). 73-78.

53. Birge, R. J., Louveaux, F., 1997. Introduction to Stochastic Programming. Springer-Verlag, New York.

54. Ramezani, M., Bashiri, M., Tavakkoli-Moghaddam, R., A new multi-objective stochastic model for a forward/reverse logistic network design with responsiveness and quality level. Applied Mathematical Modelling 37 (1) (2013), 328-344. 